\title{
Analysis of Blind Adaptive Multiuser Detection Receivers
}

\author{
${ }^{1}$ R. Nirmaladevi and ${ }^{2} \mathrm{~K}$. Kishan Rao \\ ${ }^{1}$ Dept. of Electronics \&Instrumentation Engineering, KITS Warangal, Andhra Pradesh, INDIA. \\ ${ }^{2}$ Dept. of Electronics \& Communication Engineering, Vaagdevi Group of institutions, Andhra Pradesh, \\ INDIA. \\ 1nimala123@yahoo.com, 2prof_kkrao@rediffmail.com
}

\begin{abstract}
In this paper, blind adaptive multiuser detection with Mean Output Energy (MOE) are designed in receivers for synchronous MC-DS-CDMA systems in Rayleigh fading channels. Pre and post detection in multiuser detectors are implemented in the system to detect their effects in the outputs. On the basic of the delayed received signals, we proposed Affine Projection Algorithm-link multiuser detector (APA) and its performance is evaluated with the existing multiuser blind detectors (Normalized blind LMS and Kalman Filter). Compared to existing multiuser detectors APA-link detector outperformed the BER performance and provided a tradeoff between performance and computation cost. The analysis if further extended by implementing, decorrelating multiuser detector based receivers in SC-DS-CDMA and MC-DS-CDMA to eliminate the MAI caused by other users.
\end{abstract}

Keywords: MC-DS-CDMA, MOE, APA, BER, MAI, SC-DS-CDMA

\section{Introduction}

Unlike the adaptive MMSE receivers, blind adaptive interference suppression receivers do not require training sequences. It has been shown in the original work by Honig et al. [1] that, by knowing only the spreading code and the timing of the desired user, the MMSE multiuser receiver can be implemented blindly. They have developed the first blind LMS-based multiuser detection receiver in the absence of multi-path fading. It is based on the minimization of a MAl criterion by using a stochastic gradient approach. However, this method is not convenient in a time-varying environment. For this reason, other approaches have been proposed. (i) On the one hand, blind detectors based on Recursive Least Square RLS [2] and Kalman filter [3] algorithms make it possible to improve the convergence features and tracking capabilities in a dynamic environment and interferers. (ii) On the other hand, Mucchi et al. [4] have proposed a derived version of the pioneering blind LMS-based detector, which makes it possible to operate in a time-varying frequency-selective multi-path fading channels. For this purpose, they first completed channel compensation and time alignment on the signal replicas along each independent path and then combine the resulting signals before or after multiuser detection, resulting in two receiver schemes. The first scheme is called the RAKE blind adaptive multiuser detection receiver where combining is performed after multiuser detection. The second scheme is called pre-detection combining blind adaptive multiuser detection receiver where combining is performed before multiuser detection. The pre-detection combining based receiver has the advantage of using only one detector for the 
R. Nirmaladevi and K. Kishan Rao; Analysys of Blind Adaptive Multiuser Detection Receivers, Transactions on Machine Learning and Artificial Intelligence, Volume 2 No 6 Dec, (2014); pp: 1-16

combined replicas instead of one detector for each signal replica. In addition, according to [4], this yields a remarkable complexity reduction, more reliable decision variable and more robust convergence procedure.

However, the above blind adaptive multiuser detection techniques were only developed for singlecarrier DS-CDMA systems. In this work, our purpose is to design blind adaptive multiuser detection receivers for synchronous MC-DS-CDMA systems in Rayleigh fading channels. For this purpose, we first reformulate the ideas presented in [4] to design two blind adaptive receivers for MC-DS-CDMA systems. Namely:

- The first receiver provides a blind adaptive multiuser detector for each carrier followed by a postdetection combiner.

- The second receiver consists of a pre-detection combiner followed by a single blind adaptive multiuser detector.

To implement them, we have proposed a blind APA-like multiuser detector [5]. The proposed detector can be seen as a generalization of the blind LMS-based detector [1], on the basis of multiple delayed input signal vectors. A comparative study is then carried out with existing blind LMS [1] and Kalman filter [3] based multiuser detectors initially developed for single-carrier DS-CDMA systems.

\subsection{Receiver Structure with Post-Detection Combining}

In this subsection, we propose a blind adaptive multiuser receiver with post-detection combining for synchronous MC-DS-CDMA systems in time-varying fading channels (see Figure 2.1). Thus, to retrieve the symbol sequence of the first user $d_{1}(n)$ we first recall the $N \times 1$ discrete-time received vector over the $m^{\text {th }}$ carrier given as follows:

$$
X_{m}(n)=\sqrt{P_{1}} d_{1}(n) h_{m}(n) c_{1}+\sum_{k=2}^{K} \sqrt{P}_{k} d_{k}(n) h_{m}(n) c_{k}+\eta_{m}(n)
$$

Here, as our goal is to suppress the MAl, we assume that the fading processes $\left\{h_{m}(n)\right\} m=1,2, \ldots, m$ are available at the receiver. Thus, channel compensation over the $m^{\text {th }}$ carrier can be performed in the following manner:

$$
\begin{aligned}
\mathbb{X}_{m}(n)= & \operatorname{Re}\left(h_{m}^{*}(n) X_{m}(n)\right) \\
& =\sqrt{P_{1}} d_{1}(n)\left|h_{m}(n)\right|^{2} c_{1}+\sum_{k=2}^{K} \sqrt{P_{k}} d_{k}(n)\left|h_{m}(n)\right|^{2} c_{k}+\operatorname{Re}\left(h_{m}^{*}(n) \eta_{m}(n)\right)
\end{aligned}
$$

where the multiplication with $h_{m}^{*}(n)=\left|h_{m}(n)\right| e^{-j \phi_{m}^{(n)}}$ compensates for the phase and weights the signal amplitude by a positive time-varying factor $\left|h_{m}(n)\right|^{2}$.

After channel compensation, the resulting vector $\underline{X}_{m}(n)$ over the $m^{\text {th }}$ carrier defines the input to a blind adaptive multiuser detector whose canonical linear representation for user 1 was firstly established in [1], as follows: 


$$
W_{m}(n)=c_{1}+a_{m}(n)
$$

subject to the constraint:

$$
c_{1}^{T} a_{m}(n)=0
$$

or equivalently, since $c_{1}^{T} c_{1}=1$ :

$$
c_{1}^{T} c_{1}=1
$$

Where $c_{1}$ is the normalized spreading vector of the first user and $a_{m}(n)$ is the adaptive part of the detector. Thus, the practical implementation of this detector is achieved by means of two orthogonal filters (see Figure 2): the spreading code of the desired user $c_{1}$ and the adaptive part $a_{m}(n)$ that is used to eliminate the MAI.

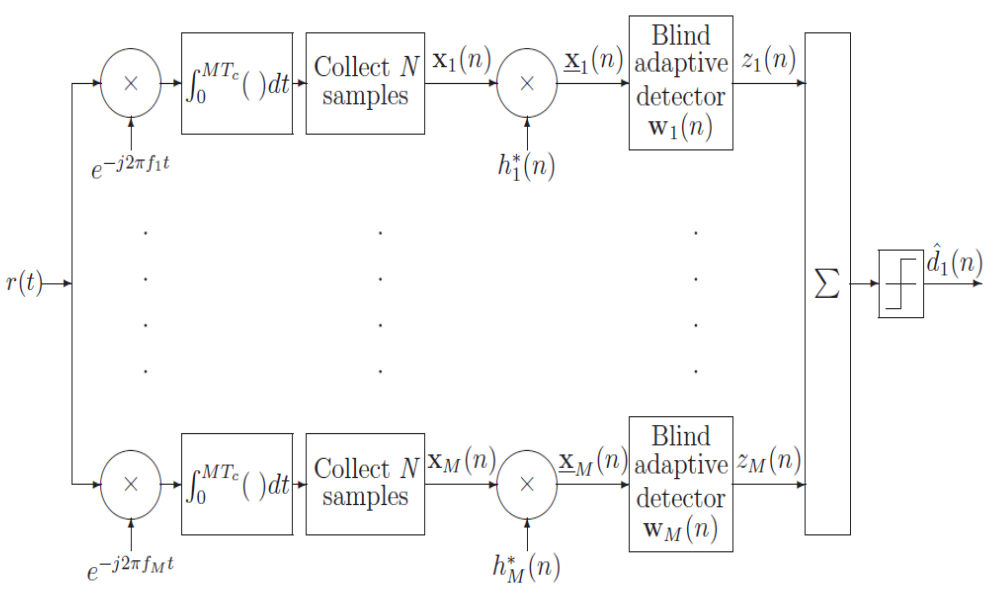

Figure 1: Blind adaptive receiver structure with post-detection combining.

The detector $W_{m}(n)$ is designed to minimize the Mean Output Energy (MOE) cost function:

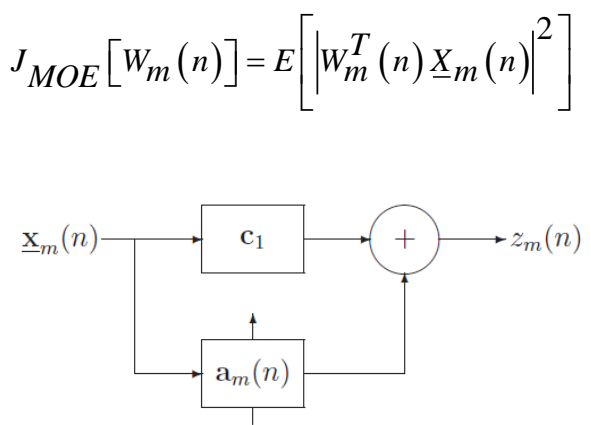

Figure 2: Blind Adaptive Multiuser Detector $W_{m}(n)=c_{1}+a_{m}(n)$.

It should be noted that the MOE criterion given by (6) is related to a "scaled" version of the MSE criterion given as follows: 


$$
\begin{aligned}
J_{M S E}\left[W_{m}(n)\right] & =E\left[\left|{\sqrt{P_{1}}}_{1}(n)-W_{m}^{T}(n) \underline{X}_{m}(n)\right|^{2}\right] \\
& =J_{M O E}\left[W_{m}(n)-P_{1}\right]
\end{aligned}
$$

Where it is assumed that $E\left[\left|h_{m}(n)\right|^{2}\right]=\sigma_{h}^{2}=1$. As $P_{1}$ is a constant, minimizing the MOE criterion will also minimize the MSE criterion. Hence, the MOE multiuser detector is equivalent to the MSE based one [1]. Since the MOE criterion does not depend on the data symbol $d_{1}(n)$, there will be no need for training sequences. Therefore, this leads to the blind adaptive implementations that will be presented in subsection [1.2]

The outputs of the blind adaptive detectors over all carriers are finally combined by a post-detection combiner resulting in the following decision about the desired user data symbol:

$$
\hat{d}_{1}(n)=\operatorname{sgn}\left(\sum_{m=1}^{M} W_{m}^{T}(n) \underline{X}_{m}(n)\right)
$$

In the next subsection, as the post-detection based receiver structure requires $M$ blind adaptive multiuser detectors, we propose a blind adaptive receiver based on pre-detection combining which requires only one multiuser detector.

\subsection{Receiver Structure with Pre-Detection Combining}

The proposed blind adaptive MOE receiver with pre-detection MRC is shown in Figure 2.3. Thus, after channel compensation and time alignment, the resulting vectors $\left\{\underline{X}_{m}(n)\right\}_{m=1,2, \ldots, M}$ given by (2) are combined before detection as follows:

$$
\underline{X}_{t o t}(n)=\sum_{m=1}^{M} \underline{X}_{m}(n)
$$

The combined vector $\underline{X}_{t o t}(n)$ is then processed with a single blind adaptive multiuser detector $W(n)=c_{1}+a(n)$ as in (3), in order to minimize the following MOE criterion:

$$
J_{M O E}[W(n)]=E\left[\left|W^{T}(n) \underline{X}_{t o t}(n)\right|^{2}\right]
$$

Where $a(n)$ is the adaptive part of the detector which satisfies $c_{1}^{T} a(n)=0$. Finally, the symbol decision of the desired user can be obtained as follows:

$$
\hat{d}_{1}(n) \operatorname{sgn}\left(W^{T}(n) \underline{X}_{t o t}(n)\right)
$$

Where the detector $W(n)$ will be implemented adaptively in the next subsection.

It should be noted that the pre-detection combining receiver structure has the advantage of reducing greatly the computational cost by using only one detector for the combined vector instead of a detector for each carrier. 


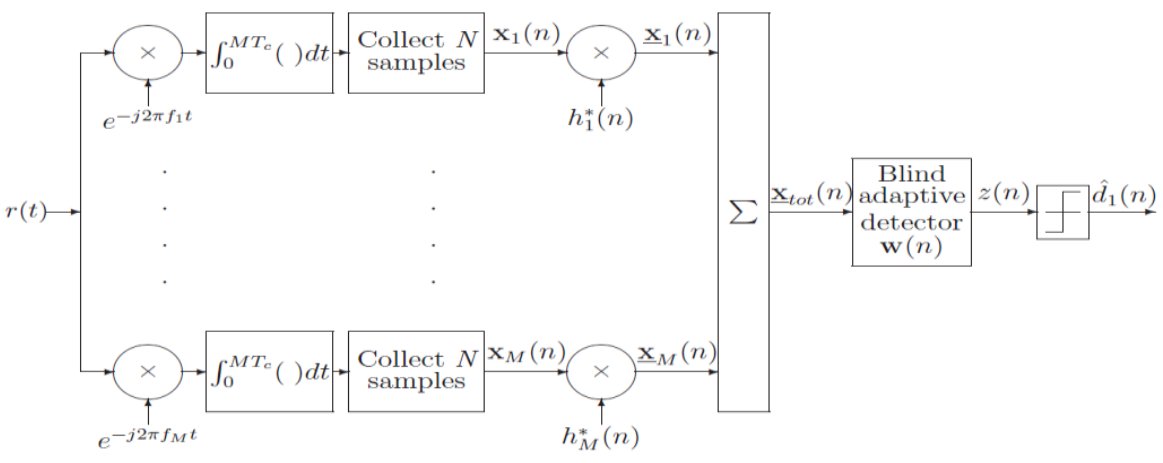

Figure 3: Blind adaptive receiver structure with pre-detection combining.

To implement the post-detection and predetection based blind adaptive receivers, various blind adaptive algorithms are considered in the next subsection.

\subsection{Blind Adaptive Implementation}

To use unique notations, let $\underline{X}(n)$ and $W(n)$ respectively denote:

- The received vector $\underline{X}_{m}(n)$ and the filter weights when considering the post- detection receiver structure.

- The combined received vector $\underline{X}_{\text {tot }}(n)$ and the filter weights $W(n)$ when using the pre-detection receiver structure.

\subsubsection{Normalized Blind LMS Based Multiuser Detector}

By using a stochastic gradient approach, Honig et al. [1] have proposed to derive an adaptive implementation for the detector (3) that minimizes the MOE criterion (6). Thus, they first evaluated the unconstraint gradient of the MOE cost function (6) as follows:

$$
\nabla J_{M O E}[W(n)]=\nabla E\left[\left|W^{T}(n) \underline{X}(n)\right|^{2}\right]=2\left[W^{T}(n) \underline{X}(n)\right] \underline{X}(n)
$$

The projected gradient, orthogonal to $\mathrm{c} 1$ is then obtained:

$$
\nabla J_{M O E}[W(n)]_{c_{1}}=2\left[W^{T}(n) \underline{X}(n)\right]\left[\underline{X}(n)-\left(c_{1}^{T} \underline{X}(n)\right) c_{1}\right]
$$

Therefore, a stochastic gradient blind algorithm to update the adaptive part of the detector can be written as follows:

$$
\begin{aligned}
a(n) & =a(n-1)-\mu \nabla J_{M O E}[W(n-1)]_{C_{1}} \\
& =a(n-1)-\mu z(n)\left[\underline{X}(n)-z_{M F}(n) c_{1}\right]
\end{aligned}
$$

Where $z(n)=W^{T}(n-1) \underline{X}(n)$ the output of the detector is, $z_{M F}(n)=c_{1}^{T} \underline{X}(n)$ is the output of the conventional matched-filter, and $\mu$ is the step-size that controls the adaptation speed. 
R. Nirmaladevi and K. Kishan Rao; Analysys of Blind Adaptive Multiuser Detection Receivers, Transactions on Machine Learning and Artificial Intelligence, Volume 2 No 6 Dec, (2014); pp: 1-16

To avoid the gradient noise amplification problem, a normalized version of this algorithm (as the NLMS algorithm) can be written as follows:

$$
a(n)=a(n-1)-\frac{\mu_{N}}{\delta+\|\underline{X}(n)\|^{2}} Z(n)\left[\underline{X}(n)-z_{M F}(n) c_{1}\right]
$$

Where $\mu_{N} \in(0,2)$ is the normalized step-size and $\delta$ is a small positive regularization constant that insures stability when $\|\underline{X}(n)\|^{2}$ is too small.

\subsubsection{Blind APA-like Multiuser Detector}

To improve the convergence features in high MOE environments and time-varying fading scenarios, we propose to generalize the algorithm in (15) by using $L$ delayed input signal vectors [5]. Toward this end, we first define the following received and code matrices of $L$ column each:

In addition, the MOE cost function in (6) is modified to account for $L$ delayed input signal vectors:

$$
\begin{aligned}
& X(n)=[\underline{X}(n) \quad \underline{X}(n-1) \ldots \quad \underline{X}(n-L+1)] \\
& C_{1}=\left[\begin{array}{llll}
c_{1} & c_{1} & \cdots & c_{1}
\end{array}\right] \\
& J_{M O E}[W(n)]=E\left[\left\|X^{T}(n) W(n)\right\|^{2}\right]
\end{aligned}
$$

Taking the unconstraint gradient of the MOE cost function:

$$
\nabla J_{M O E}[W(n)]=\nabla E\left[\left\|X^{T}(n) W(n)\right\|^{2}\right]=2 X(n)\left[X^{T}(n) W(n)\right]
$$

The projected gradient, orthogonal to $C_{1}$ satisfies:

$$
\nabla J_{M O E}[W(n)]_{C_{1}}=2\left[X(n)-C_{1}\left(C_{1}^{T} X(n)\right)\right]\left[X^{T}(n) W(n)\right]
$$

Then, a stochastic gradient algorithm that updates the adaptive part of the detector (3) can be written as follows:

$$
a(n)=a(n-1)-\mu \nabla J_{M O E}[W(n-1)]_{c_{1}}
$$

Where $\mu$ is the step-size.

Substituting (20) in (21) and introducing a factor $\left[\delta I_{L}+X^{T}(n) X(n)\right]^{-1}$ similar to that in the APA, a new blind algorithm (APA-like) to update $a(n)$ can be expressed by [5]:

$$
a(n)=a(n-1)-\mu_{N}\left[X(n)-C_{1} Z_{M F}(n)\right]\left[\delta I_{L}+X^{T}(n) X(n)\right]^{-1} z(n)
$$

Where $z(n)=X^{T}(n) w(n-1), Z_{M F}(n)=C_{1}^{T} X(n), \mu_{N} \in(0,2)$ and $\delta$ is the regularization constant. 
To insure that the orthogonally condition $c_{1}^{T} a(n)=0$ is satisfied at each iteration, we replace $a(n)$ by its orthogonal projection onto $c_{1}$ :

$$
a(n)=a(n)-c_{1}^{T} a(n) c_{1}
$$

It should be noted that when $L=1$, the blind APA-like multiuser detector (22) reduces to the normalized blind LMS multiuser detector (15).

\subsubsection{Blind Multiuser Detection Based on Kalman Filtering}

In [3], Zhang et al. have proposed to use an alternative standard representation for the blind adaptive multiuser detector:

$$
W(n)=c_{1}-A_{1} a(n)
$$

Where the columns of the $N \times N-1$ matrix $A_{1}$ span the null space of $c_{1}$, i.e.:

$$
c_{1}^{T} A_{1}=0
$$

It should be noted that $A_{1}$ can be precomputed off-line via one of many orthogonalization procedures such as the Gram-Schmidt orthogonalization. Unlike (3), the adaptive part $a(n)$ in (24) is now of size $(N-1) \times 1$ and has the advantage of being unconstraint.

Let us define the output of the detector as follows:

$$
z(n)=W^{T}(n) \underline{X}(n)
$$

then $z(n)$ has zero-mean and its variance is given by (due to equations (6) and (7):

$$
E[|Z(n)|]^{2}=J_{M O E}[W(n)]=J_{M S E}[W(n)]+P_{1}
$$

Thus, when the detector is optimal (i.e., $J_{M S E}[W(n)]$ attains its MMSE value), the variance of $z(n)$ corresponds to the minimum MOE and is dominated by the power of the desired user $P_{1}$.

Substituting (24) in (26) results in the following measurement equation:

$$
Z_{M F}(n)=\underline{d}^{T}(n) a(n)+Z(n)
$$

Where $Z_{M F}(n)=c_{1}^{T} \underline{X}(n)$ and $\underline{d}^{T}(n)=\underline{X}^{T}(n) A_{1}$.

If the detector is assumed to be time-invariant, one can write:

$$
a(n)=a(n-1)
$$

As (28) and (29) define a state-space representation of the adaptive part of the detector, Kalman filtering makes it possible to recursively update $a(n)[3]$.

\subsubsection{Computational Cost of the Various Algorithms}

Here, we provide the computational cost of the various blind adaptive multiuser detectors when used to implement the post-detection and pre-detection combining receiver structures. According to Table 1, 
R. Nirmaladevi and K. Kishan Rao; Analysys of Blind Adaptive Multiuser Detection Receivers, Transactions on Machine Learning and Artificial Intelligence, Volume 2 No 6 Dec, (2014); pp: 1-16

the predetection combining receiver structure with the Table 1: Computational complexity of the various blind adaptive multiuser detectors when considering the post-detection and predetection combining receiver structures.

Table: 1

\begin{tabular}{|c|c|c|}
\hline $\begin{array}{c}\text { Blind Adaptive } \\
\text { Detector }\end{array}$ & $\begin{array}{c}\text { Post-detection } \\
\text { Combining }\end{array}$ & $\begin{array}{c}\text { Pre-detection } \\
\text { Combining }\end{array}$ \\
\hline LMS based detector & $O(M N)$ & $O(N)$ \\
\hline APA-like Detector & $O\left(M N L^{2}\right)$ & $O\left(N L^{2}\right)$ \\
\hline $\begin{array}{c}\text { Kalman Based } \\
\text { Detector }\end{array}$ & $O\left(M N^{2}\right)$ & $O\left(N^{2}\right)$ \\
\hline
\end{tabular}

Various blind adaptive detectors has the advantage of greatly reducing the computational cost when compared with the post-detection combining based one. In addition, the proposed blind APA-like detector has the advantage of providing a scalable complexity by adjusting the parameter $L$ which is usually much less than the filter length (i.e., $L<<N$ ). The scalable complexity of the APA-like detector can be traded with the performance as we will see in the simulation results presented in the next subsection. Therefore, the APA-like detector with the pre-detection combining scheme is a priori preferable to design the receiver.

\section{Simulation Results}

In this subsection, we first carry out a comparative study between the following blind adaptive multiuser detectors:

- The normalized version of the standard blind LMS multiuser detector [1],

- The proposed blind APA-like multiuser detector,

- The blind Kalman filter based multiuser detector [3], when they are used to implement the predetection and post-detection combining receiver structures.

In addition, we compare the performances of the proposed blind APA-like detector with the training based APA filter presented in subsection III.

A synchronous MC-DS-CDMA system with $\mathrm{K}$ active users and $M=4$ carriers is considered. The spreading sequences used are gold codes of length $N=31$. The fading processes $\left\{h_{m}(n)\right\}_{m}=1,2, \ldots$, Mare generated according to the complex Gaussian distribution with zero-mean and unit-variance. User 1 is assumed to be the desired user with SNR per transmitted carrier kept constant at $10 \mathrm{~dB}$.

In the first example, a high MAl scenario is assumed with 14 multiple-access interfering users (i.e., $K=15$ ), among which five users have ISR (see equation 1.50) of $10 \mathrm{~dB}$ each, five users have ISR of $20 \mathrm{~dB}$ each, two users have ISR of $30 \mathrm{~dB}$ each and two other users have ISR of $40 \mathrm{~dB}$ each. According to Figure 2.4, the pre-detection combining receiver slightly outperforms the post-detection receiver with the various blind adaptive detectors, when considering the BER performance. In addition, the proposed blind APA-like detector provides much better BER performance and convergence features than the normalized blind LMS detector. Nevertheless, the blind Kalman detector yields the best BER performance and convergence features, but at the price of increased computational cost (see Table 2.1). 
Figure 2.5 demonstrates the average Signal to Interference- plus-Noise Ratio (SINR) performance of the various blind detectors in the pre-detection combining receiver. The average SINR is defined as follows:

$$
\operatorname{SINR}(n)=\frac{\sum_{l=1}^{\text {loop }}\left|W^{T}(n)\left(\pi \sqrt{P_{1}} d_{1}(n) c_{1, t o t}\right)\right|^{2}}{\sum_{l=1}^{\text {loop }}\left|W^{T}(n)\left[\underline{X}(n)-\sqrt{P_{1}} d_{1}(n) c_{1, t o t}\right]\right|^{2}}
$$

Where

$$
c_{1, t o t}=\sum_{m=1}^{M}\left|h_{m}(n)\right|^{2} c_{1}
$$

and the average is performed over loop $=300$. The SINR improvement of the proposed APA-like detector is better than that obtained with the normalized blind LMS detector and approaches that of the blind Kalman detector, when $L$ is getting higher. Therefore, for the various reasons mentioned above, we recommend to use the blind APA- like detector with the pre-detection combining scheme to design the receiver.

To illustrate the advantages of the blind adaptive MOE detectors over the training based adaptive MMSE filters presented in the previous section, we consider a high MAI scenario with 8 interfering users for which the ISR $=20 \mathrm{~dB}$ (this hence corresponds to a severe near-far scenario). Figure 6 shows the SINR performance improvement of the proposed APA-like detector and the training based APA filter in the pre-detection combining receiver. According to this figure, the blind detector yields better SINR performance improvement than the training based filter which suffers from slow convergence in this severe near-far scenario. This is due to the fact that the blind detector uses the code sequence of the desired user in addition to an adaptive part, whereas the training based filter starts adaptation from a zero initial weight vector. Thus, in a high MAl environment, it is recommended to use a blind adaptive detector to efficiently suppress the MAI and to mitigate the near-far problem.

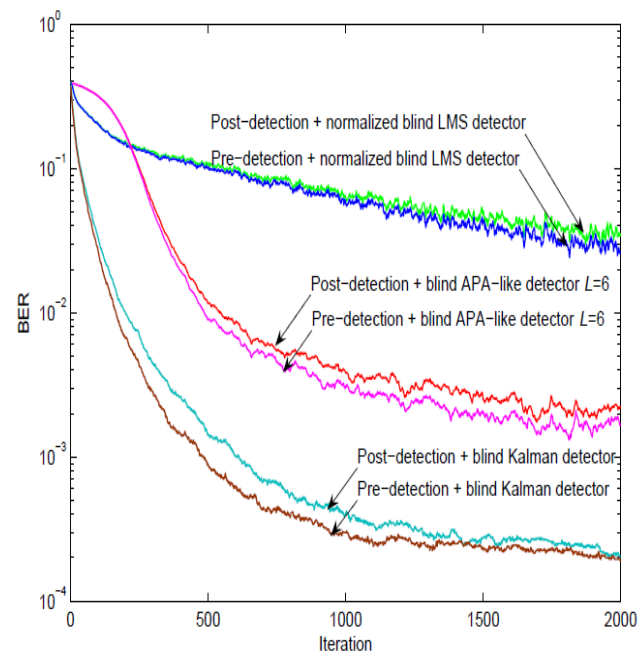

Figure 4: BER performance of the pre-detection and post-detection receivers with the various blind adaptive multiuser detectors. 


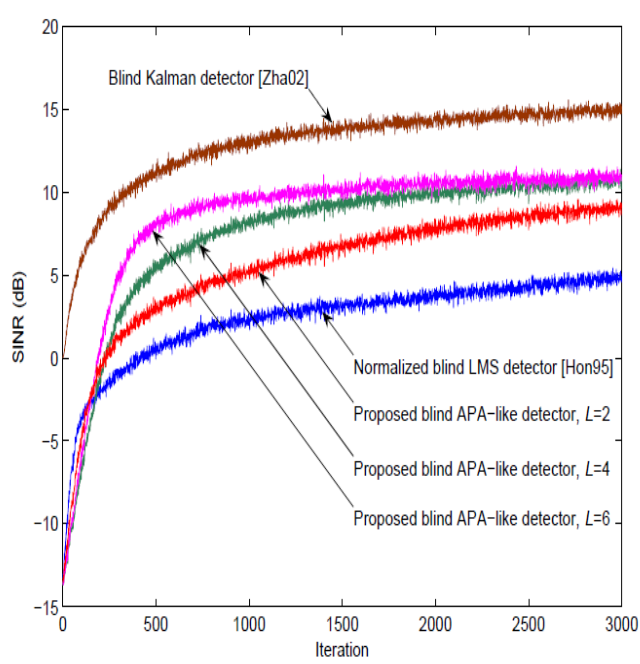

Figure 5: SINR improvement of the various blind adaptive detectors in the predetection combining receiver.

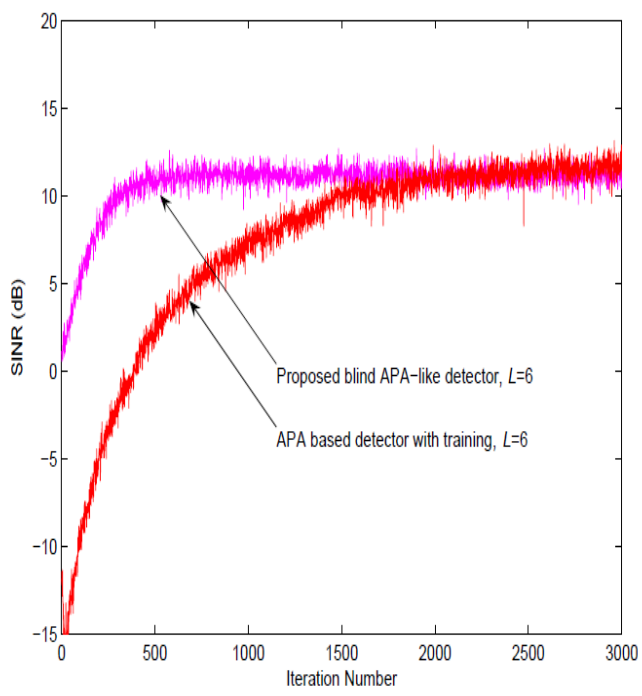

Figure 6: SINR improvement of the proposed blind APA-like detector and the training based APA filter in the pre-detection combining receiver.

\section{Receiver Design Based on Decorrelation Detection}

As an alternative to the MMSE multiuser receivers, the decorrelating multiuser detector based receivers are designed to completely eliminate the MAl caused by other users, using the spreading sequences of all users [7]. In this section, we first present the state of the art on single-carrier DS-CDMA receiver design based on the decorrelating detector. Then, we propose a MC-DS-CDMA receiver structure consisting of a decorrelating detector, a Kalman filter based channel estimator and a MRC [6].

\subsection{Decorrelation detection for single-carrier DS-CDMA}

Given the spreading codes of all active users $\left\{c_{k}\right\}_{k=1, \ldots, \mathrm{K}}$ the decorrelating multiuser detector for user 1 can be written in the following form [8]:

$$
W_{1}=\sum_{k=1}^{K}\left[R^{-1}\right] 1 k c_{k}
$$

Where $R=\left[\begin{array}{llll}c_{1} & c_{2} & \cdots & c_{k}\end{array}\right]^{T}\left[\begin{array}{llll}c_{1} & c_{2} & \cdots & c_{k}\end{array}\right]$ the normalized cross-correlation matrix of the spreading is vectors and $\left[R^{-1}\right]_{i j}$ denotes the $(i, j)^{\text {th }}$ element of the inverse of the matrix $R$.

Such decorrelating multiuser detector satisfies:

$$
W_{1}^{T} c_{1}=1
$$

and

$$
W_{1}^{T} c_{k}=0, \quad k=2, \ldots, K
$$


Equations (33) and (34) can be verified as follows:

$$
\begin{aligned}
W_{1}^{T} c_{k} & =\sum_{i=1}^{K}\left[R^{-1}\right]_{1 c_{i}^{T} c_{k}} \\
& =\sum_{i=1}^{K}\left[R^{-1}\right]_{1 i}[R]_{i k} \\
& =\left[R^{-1} R\right]_{1 k} \\
& =[I]_{1 k} \\
& = \begin{cases}1 & k=1 \\
0 & k=2, \ldots, \mathrm{K}\end{cases}
\end{aligned}
$$

It is obvious from (34) that the decorrelating detector is orthogonal to the subspace spanned by the spreading sequences of all interfering users. Therefore, the decorrelating detector can completely eliminate the MAI and can achieve optimal near-far resistance whatever the ISR of the interfering users. It should be emphasized that the decorrelating detector does not require the knowledge of the power of all users.

The decorrelating detector based receiver for synchronous DS-CDMA systems is first proposed by Lupas and Verd' $u$ [9]. The generalization to asynchronous DS-CDMA systems is then reported in [10]. However, only AWGN channels are considered. Since then, the extension of these receivers to operate in fading channels has been extensively studied by several authors. Thus, Zvonar et al. [11] have analyzed the performance of the decorrelating detector in slowly time-varying flat-fading channels. Nevertheless, the fading processes are assumed to be available at the receiver. In [12], Kawahara et al. have proposed to combine decorrelation multiuser detection with channel estimation for asynchronous DS-CDMA systems in multi-path slowly fading channels. The fading processes are estimated by using a training based RLS algorithm. In [13], a decorrelating detector based receiver structure is considered for application in rapidly time-varying Rayleigh fading channels where the fading processes are estimated adaptively using the LMS or the RLS algorithm. Recently, in [14], Wu et al. have compared the performances of a Kalman filter based channel estimator combined with various multiuser detectors, such as the decorrelating detector, the decision-feedback detector, the parallel and successive interference cancelation. According to their study, the decorrelating detector is the most robust detector against the MAI and the near-far problem.

\subsection{MC-DS-CDMA receiver based on decorrelation detection}

Although decorrelation multiuser detection have been extensively used for single-carrier DS-CDMA systems, few approaches are developed for MC-DS-CDMA systems. Thus, Yang et al. [15] have proposed several decorrelation multiuser detection schemes for TF-domain spreading MC-DS-CDMA systems in AWGN channels. However, they did not investigate these schemes in fading channels. In [16], the authors have developed a multichannel joint detection scheme for MC-DS-CDMA systems in timeinvariant frequency-selective fading channels. The scheme consists of a decorrelating detector followed by a RAKE multi-path combiner for each carrier. Nevertheless, perfect channel knowledge is assumed at the receiver.

Here, we propose to extend, to the multi-carrier case, the combination of decorrelation multiuser detection and Kalman channel estimation scheme presented in [14]. In particular, our scheme [6] operates in three steps (see Figure 7): 
R. Nirmaladevi and K. Kishan Rao; Analysys of Blind Adaptive Multiuser Detection Receivers, Transactions on Machine Learning and Artificial Intelligence, Volume 2 No 6 Dec, (2014); pp: 1-16

1. The decorrelating multiuser detector is carried out along each carrier to completely eliminate the $\mathrm{MAl}$,

2. The fading channel responses, modeled by Auto Regressive (AR) processes, are estimated by using Kalman filtering,

3. The fading processes estimates are fed into a frequency diversity MRC rule to obtain the data symbol estimate.

Thus, to retrieve the desired symbol sequence of the first user $d_{1}(n)$ from the received signal, let us recall the $N \times 1$ discrete time received vector over the $m^{\text {th }}$ carrier given as follows:

$$
X_{m}(n)=\sqrt{P_{1}} d_{1}(n) h_{m}(n) c_{1}+\sum_{k=2}^{K} \sqrt{P_{k}} d_{k}(n) h_{m}(n) c_{k}+\eta_{m}(n)
$$

Where the fading processes $\left\{h_{m}(n)\right\}_{m}=1,2, \ldots, M$ are assumed to be rapidly time-varying. At that stage, the received vector at the $m^{\text {th }}$ carrier $X_{m}(n)$ is processed by the decorrelating detector $W_{1}$ given by (32).

By taking into account (33) and (34), the decorrelating detector yields the following observation:

$$
y_{m}(n)=W_{1}^{T} X_{m}(n)=\sqrt{P_{1}} d_{1}(n) h_{m}(n)+v_{m}(n)
$$

Where $v_{m}(n)=W_{1}^{T} \eta_{m}(\eta)$ is a zero-mean Gaussian noise with variance $\sigma_{v}^{2}=\sigma_{\eta}^{2}\left[R^{-1}\right]_{11}$. From equation (37), the decorrelating detector is able to completely eliminate the MAl caused by other users, but at the expense of slightly enhancing the additive noise.

Based on the observations $\left\{y_{m}(n)\right\}_{m=1, \ldots, M}$ and by using an AR model for the fading processes, Kalman filtering can be carried out to provide an estimation of the fading processes $\left\{\hat{h}_{m}(n)\right\}_{m=1,2, . ., M}[6]$.

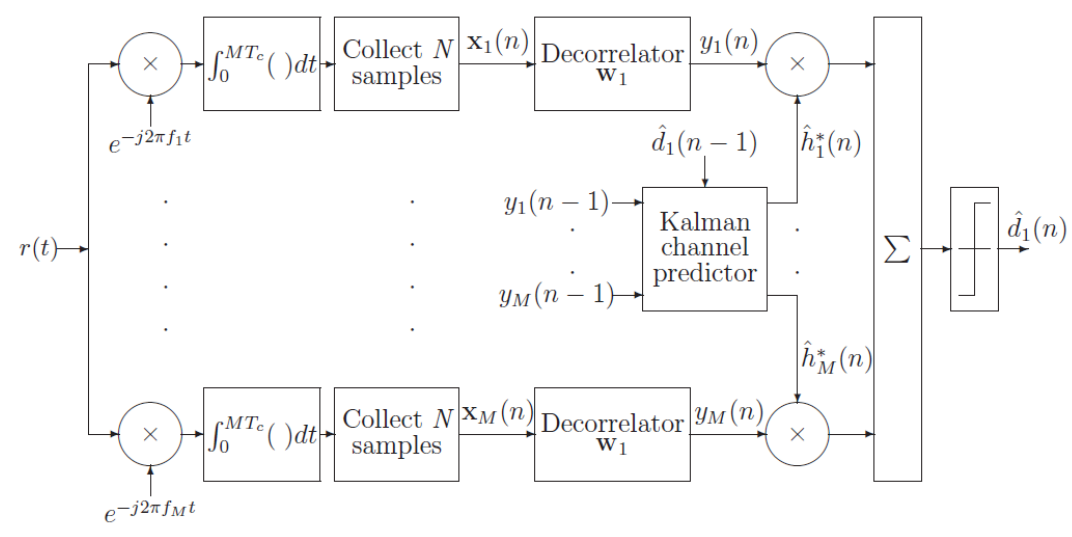

Figure 7: MC-DS-CDMA receiver structure with decorrelation detection for user 1. 
The estimation of AR fading channels based on Kalman filtering will be investigated in chapter 3 . This also includes the development of several channel estimation techniques that can be directly applied to the proposed receiver in Figure 7.

Finally, MRC makes it possible to provide the estimate of the desired user data symbol as follows:

$$
\hat{d}_{1}(n)=\operatorname{sgn}\left(\operatorname{Re}\left(\sum_{m=1}^{M} h_{m}^{*}(n) y_{m}(n)\right)\right)
$$

\subsection{Simulation results}

In this subsection, we first carry out a comparative simulation study between the proposed decorrelating detector based receiver and the correlator based one presented in [15].

A synchronous MC-DS-CDMA system is considered with $M$ carriers and $K$ active users, each using a gold code of length $N=31$ to spread his information. The fading processes $\left\{h_{m}(n)\right\}_{m=1,2, . ., M}$ are generated according to the modified Jakes model with $L_{O}=16$ oscillators and Doppler rate $f_{d} T_{b}=0.05$. Here, to focus our attention on the effect of the MAI on both receivers, the fading processes are assumed to be available at the receiver. The performance of the decorrelating detector based receiver when the fading processes are estimated by various approaches will be presented in the simulation results.

Figure 8 shows the effects of the ISR on the BER performance of both receivers for number of carriers equal to $M=1$ and $M=3$. On the one hand, the BER of the decorrelating detector based receiver does not depend on the ISR and, hence, it is near-far resistant. On the other hand, the BER of the correlator detector based receiver is highly dependent on the ISR, where degradation of the BER can be noticed starting at low ISR. In addition, for the decorrelating detector based receiver, a high frequency diversity gain is obtained when increasing the number of carrier from $M=1$ to $M=3$. This is not the case for the correlator based receiver when the ISR is high.

According to Figure 9, increasing the number of users greatly increases the BER of the correlator based receiver. On the other hand, the decorrelating detector based receiver is insensitive to the number of users. This is due to the fact that, contrary to the correlator based receiver, the decorrelating detector based receiver can completely eliminate the MAl caused by other users.

Here, we also present a simulation example that illustrates the performance of the proposed decorrelating detector based receiver compared with that of the adaptive receivers proposed in the previous sections. Namely, the training based Separate Detection (SD) receiver with APA and the postdetection combining receiver with blind APA-like detector. To focus on the MAI suppression capabilities of these receivers, we consider only AWGN channels (without the effect of fading) with $K=10, M=3$, $S N R=5 \mathrm{~dB}$ and ISR=15 dB.

According to Figure 10, the decorrelating detector based receiver provides the lowest BER results without the need for any training period. This is due to the fact that it uses the spreading codes of all users and, hence, can completely eliminate the MAI. The blind APA based receiver has faster convergence than the training APA based one, but it results in higher steady state BER. To take the advantages of both adaptive receivers, their combination can also be considered. Thus, the blind APA- 
R. Nirmaladevi and K. Kishan Rao; Analysys of Blind Adaptive Multiuser Detection Receivers, Transactions on Machine Learning and Artificial Intelligence, Volume 2 No 6 Dec, (2014); pp: 1-16

like detector is first carried out up to iteration number 500. As the blind APA-like detector uses the spreading code of the desired user, this will ensure the fast suppression of high amount of MAI.

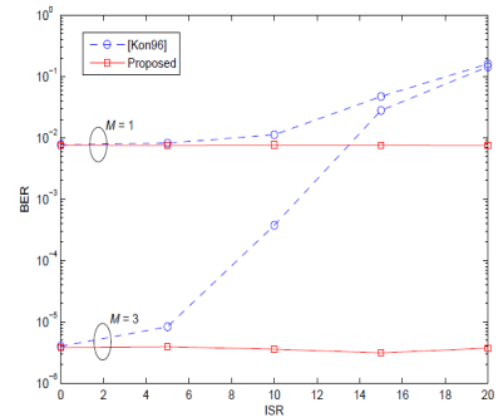

Figure 8: BER performance versus ISR for the proposed receiver and the correlator based one [Kon96]. Number of carriers considered are $M=1$ and $M=3 . K=10$ and $S N R=15 \mathrm{~dB}$.

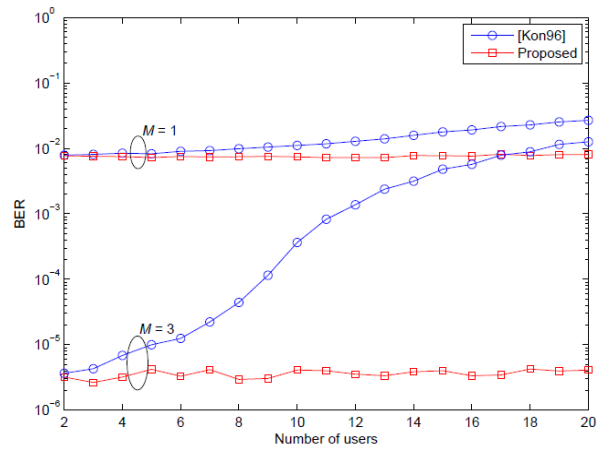

Figure 9: BER performance versus number of active users $K$ for the proposed receiver and the one in [18]. Number of carriers considered are $M=1$ and $M=3$. ISR=10 dB and SNR=15 dB.

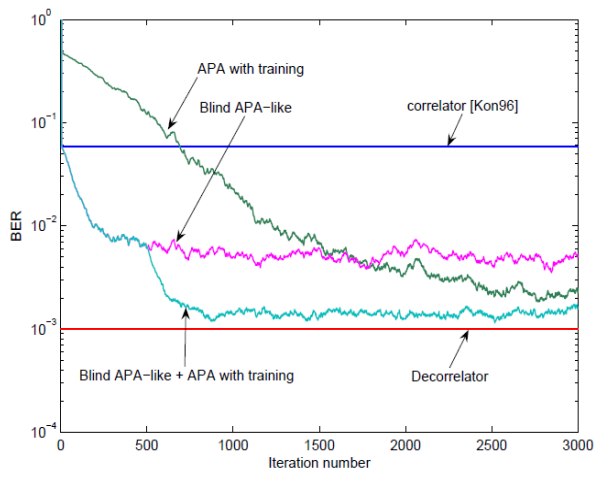

Figure 10: BER performance versus iteration number for the various receivers. $M=3, K=10, I S R=15 \mathrm{~dB}$ and SNR=5 dB.

At that stage, starting from the final filter weight values provided by the blind APA-like detector (at iteration 500), a training based APA can then be used to continue eliminating the residual MAI and can provide a BER performance close to that of the decorrelating detector based receiver.

\section{Conclusions}

In this work, we have proposed five MAI suppression receivers for MC-DS-CDMA systems in Rayleigh fading channels.

We first presented two adaptive MMSE receiver structures based on adaptive filters such as NLMS, APA and RLS. The so-called Separate Detection (SD) structure consists in considering a particular adaptive filter for each carrier, whereas the so-called Joint Detection (JD) structure is defined by the concatenation of the adaptive filter weights dedicated to each carrier. Simulation results show that the JD structure provides lower BER than the SD structure. In addition, the order of complexity of both structures are the same when considering the APA, but this is not the case for the RLS filter where the JD structure has higher computational cost than the SD one. Therefore, APA in the JD structure corresponds to a trade-off between performance and computational cost. 
However, as adaptive receivers require training sequences, we have proposed two blind adaptive Mean Output Energy (MOE) receivers based on an APA-like multiuser detector, where only the spreading sequence and the timing of the desired user are required. The so-called post-detection combining based receiver structure provides a blind adaptive detector for each carrier where combining is performed after detection. The so-called pre-detection combining based receiver structure uses a single blind adaptive detector after combining the signals of all carriers. Therefore, the computational cost of the pre-detection combining based receiver is much less than that of the post-detection combining based one. Simulation results show that the pre-detection combining based receiver provides slightly lower BER results than the post-detection combining based one. In addition, the comparative study is carried out with existing blind LMS and Kalman filter based detectors shows that the proposed APA-like detector can provide a trade-off between performance and computational cost. Furthermore, the proposed blind APA-like detector is shown to outperform the training based APA in severe near-far scenarios, when considering the BER and SINR improvement.

When the spreading codes of all active users are available, we propose a receiver structure based on the decorrelating detector which includes also a Kalman channel estimator and a MRC [6]. The comparative simulation study we have carried out shows that, while the correlator based receiver is highly sensitive to the MAI and the near-far problem, the proposed receiver can completely eliminate the MAl and is insensitive to the near-far problem. In addition, the proposed receiver can provide approximately the same BER performance as the adaptive receivers without the need of any training sequences for MAI suppression.

Nevertheless, the design of receivers usually requires the explicit estimation of the fading process over each carrier to achieve optimal diversity combining and coherent symbol detection.

\section{REFERENCES}

[1]. M. Honig, U. Madhow, and S. Verdu, "Blind adaptive multiuser detection", IEEE Trans. Inf. Theory, vol. 41, no. 7, pp.944-960 1995.

[2]. Yihai Zhang, Wu-Sheng and T.Aron Gulliver, "Recursive multi-user detection for DS-WMB system", IEEE Conf. Communications, Computers and Signal Processing (PACRM), pp- 534-537, Aug., 2005.

[3]. X. D. Zhang and W. Wei, "Blind adaptive multiuser detection based on Kalman filtering", IEEE Trans. Signal Process., vol. 50, no. 1, pp.87 -95 2002.

[4]. L. Mucchi, S. Morosi, E. Del Re and R. Fantacci, "A new algorithm for blind adaptive multiuser detection in frequency selective multipath fading channel," IEEE Trans. On wireless commun., vol. 3, no1, pp. 235-247, January 2004.

[5]. A. Carini, G.L. Sicuranza, "Optimal Regularization Parameter of the Multichannel Filtered- $x$ Affine Projection Algorithm", IEEE Transactions on Signal Processing, v.55 n.10, p.4882-4895, October 2007. 
R. Nirmaladevi and K. Kishan Rao; Analysys of Blind Adaptive Multiuser Detection Receivers, Transactions on Machine Learning and Artificial Intelligence, Volume 2 No 6 Dec, (2014); pp: 1-16

[6]. S. Alireza Banani, Rodney G. Vaughan "Blind channel estimation for MRC systems with maneuvering transmit/receive terminals", Canadian Conference on Electrical and Computer Engineering, (CCECE 2010), Calgary, Alberta, Canada, 2-5 May, 2010.

[7]. J.Ravindrababu, P.Venumadhav, E.V.Krishna Rao,"BER Performance of Multi-User MC-DSCDMA using spreading codes in fading Channels," IEEE ICCIC-2011 pp. 301-304.

[8]. S. Ulukus and R. D. Yates, "A blind adaptive decorrelating detector for CDMA systems," IEEE J. Select. Areas Commun. vol. 16, pp. 1530-1541, Oct. 1998.

[9]. R. Lupas and S. Verd'u, "Linear Multiuser Detectors for Synchronous Code Division MultipleAccess Channels" IEEE Transactions on Information Theory, vol. 35, pp. 123--136, January 1989.

[10]. Lupas R., and S. Verdd, "Near-far resistance of multiuser detectors in asynchronous channels," IEEE Trans. Commun., pp. 496-508, 1990.

[11]. Z. Zvonar, "Combined multiuser detection and diversity reception for wireless CDMA systems," IEEE Trans. Veh. Technol., vol. 45, pp. 205-211, Feb. 1996.

[12]. T. Kawahara and T. Matsumoto, "Joint decorrelating multiuser detection and channel estimation in asynchronous CDMA mobile communications channels," IEEE Trans. Veh. Technol., vol. 44, pp. 506-515, Aug. 1995.

[13]. Shakya, F.H. Ali, and E. Stipidis, "Improved successive interference cancellation for DS-CDMA using constant modulus algorithm", In International Symposium on Communications Theory and Applications, Amblseide, UK, 1-6, July 2007.

[14]. S.-H. Wu, U.Mitra and C.-C.J.Kuo, "Performance of Linear multistage receivers for Ds-CDMA in frequency Selective fading channels", IEEE Trans. Inf. Theory, Vol.51, no.10, pp-3493-3517, Oct.2005.

[15]. Lie-Liang Yang, Multicarrier Communications, John Wiley Sons, 2009. 